\title{
Recurrence of cavernous malformations after surgery in childhood
}

\author{
Laura M. Prolo, MD, PhD, ${ }^{1}$ Michael C. Jin, BS, ${ }^{1}$ Tina Loven, DO, ${ }^{3}$ Hannes Vogel, MD, ${ }^{2}$ \\ Michael S. B. Edwards, MD, ${ }^{1}$ Gary K. Steinberg, MD, PhD, ${ }^{1}$ and Gerald A. Grant, MD1 \\ Departments of ${ }^{1}$ Neurosurgery and ${ }^{2}$ Pathology, Stanford University School of Medicine, Stanford, California; and ${ }^{3}$ Department of \\ Pediatric Neurosurgery, Global Neuroscience Institute/St. Christopher's Hospital for Children, Philadelphia, Pennsylvania
}

\begin{abstract}
OBJECTIVE Cavernous malformations (CMs) are commonly treated cerebrovascular anomalies in the pediatric population; however, the data on radiographic recurrence of pediatric CMs after surgery are limited. The authors aimed to study the clinical presentation, outcomes, and recurrence rate following surgery for a large cohort of CMs in children.

METHODS Pediatric patients ( $\leq 18$ years old) who had a CM resected at a single institution were identified and retrospectively reviewed. Fisher's exact test of independence was used to assess differences in categorical variables. Survival curves were evaluated using the Mantel-Cox method.

RESULTS Fifty-three patients aged 3 months to 18 years underwent resection of 74 symptomatic CMs between 1996 and 2018 at a single institution. The median length of follow-up was 5.65 years. Patients most commonly presented with seizures $(45.3 \%, n=24)$ and the majority of CMs were cortical $(58.0 \%, n=43)$. Acute radiographic hemorrhage was common at presentation $(64.2 \%, n=34)$. Forty-two percent $(n=22)$ of patients presented with multiple CMs, and they were more likely to develop de novo lesions (71\%) compared to patients presenting with a single CM (3.4\%). Both radiographic hemorrhage and multiple CMs were independently prognostic for a higher risk of the patient requiring subsequent surgery. Fifty percent $(n=6)$ of the 12 patients with both risk factors required additional surgery within 2.5 years of initial surgery compared to none of the patients with neither risk factor $(n=9)$.

CONCLUSIONS Patients with either acute radiographic hemorrhage or multiple CMs are at higher risk for subsequent surgery and require long-term MRI surveillance. In contrast, patients with a single CM are unlikely to require additional surgery and may require less frequent routine imaging.

https://thejns.org/doi/abs/10.3171/2020.2.PEDS19543
\end{abstract}

KEYWORDS cavernoma; cavernous malformation; pediatric; recurrence rate; vascular anomaly; vascular disorders

$\mathrm{C}$ AVERNOUS malformations (CMs) are dilated capillary vessels that lack a blood-brain barrier and are prone to focal microhemorrhage and hemosiderin deposition. ${ }^{1,2}$ The sluggish blood flow associated with CMs renders them occult on angiography-thus, they are diagnosed with CT or MRI. CMs are the next most common vascular malformation to developmental venous anomalies (DVAs) and account for 10\%-15\% of all vascular malformations. ${ }^{2}$ Twenty-five percent of CMs present in childhood; ${ }^{3,4}$ however, data on the natural history of pediatric CMs after resection are limited.

The purpose of this study was to characterize the clinical course of pediatric patients who underwent resection of a $\mathrm{CM}$. We aimed to determine if clinical course and recur- rence rate differed between patients who presented with a single CM compared to those who had multiple CMs on initial MRI. Furthermore, in the group of children who had subsequent surgeries, we evaluated whether the symptomatic CMs were present on the initial MRI or whether these children developed de novo lesions that later became symptomatic. We identify risk factors predicting which patients are likely to require additional surgeries and we propose guidelines for long-term patient monitoring.

\section{Methods}

\section{Study Design and Patient Population}

This study was carried out with the approval of Stan-

ABBREVIATIONS aHR = adjusted HR; CM = cavernous malformation; DVA = developmental venous anomaly; GTR = gross-total resection; $H R=$ hazard ratio; STR = subtotal resection. 
ford's institutional review board. We retrospectively reviewed all hospital databases to find pediatric patients (defined as being 18 years of age or younger) who had either a pathologically proven $\mathrm{CM}$ or a pathology report identifying a vascular malformation, with history, imaging, and operative report describing a first CM resection between January 1996 and December 2017 at either Lucile Packard Children's Hospital or Stanford Hospital and Clinics. (One patient had a fourth resection in 2018.) Results from 8 neurosurgeons are reported in this series. Patients were drawn from a pathology database and through a systematic search of medical records conducted using ICD-9 code 747.81 (congenital anomalies of the cerebrovascular system) between birth and 18 years of age. Clinical documents were subsequently filtered using a free-text search of the keywords "cavernous" and "malformation." Only patients aged 18 years or younger at the time of initial surgery were included. Obtained records were subsequently reviewed manually to confirm pathology.

Of 113 charts reviewed, 53 patients met the criteria and were included in this study (Fig. 1). Seventeen patients were excluded because the final pathology report showed arteriovenous malformation. This surgical series focused on patients with pathologically proven CMs-thus, 43 patients who had not undergone surgery were excluded. For the 53 patients who met our criteria, we reviewed clinical records, radiological findings, operative details, and complications.

\section{Data Collection and Definitions}

We retrospectively reviewed all patient charts to determine age at initial and subsequent CM resections, sex, ethnicity, presentation, location of CM, number of and location of lesions on initial and subsequent MRI examinations, associated DVA or other vascular malformation, family history, and radiation history. We noted whether a gross-total resection (GTR) or subtotal resection (STR) was achieved. We defined a GTR as an absence of lesion based on both the operative report and postoperative imaging, whereas for STRs either the operative report and/ or postoperative MRI mentioned residual lesion. If subsequent imaging identified a CM in the same location as a previous lesion that had been totally resected, we identified the lesion as a recurrent lesion. If the previous lesion was subtotally resected, a subsequent lesion in the same location was defined as a residual lesion. A CM that was not noted on initial MRI but became apparent on subsequent MRI scans was defined as a de novo lesion. Acute hemorrhage was described in the radiology report and confirmed by us as an overt hemorrhage based on imaging (increased T1 intensity on MRI or hyperdensity on CT). Thirty-three of the 34 patients with acute hemorrhage were symptomatic: seizures $(n=14)$, neurological deficit $(n=15)$, severe headache $(n=1)$, or somnolence $(n=3)$. One patient with a family history of CMs was found to have a hemorrhagic lesion incidentally after a fall. An increase in the hemosiderin halo or the CM diameter alone was not considered acute hemorrhage. Progression was defined as enlargement of a previously resected $\mathrm{CM}$ on subsequent imaging. Rates of subsequent surgeries were defined as the number of additional surgeries divided by the total number of per- son-years of clinical follow-up. Rates of lesion recurrence were defined as the number of recurrences divided by the total number of lesion-years of radiological follow-up.

\section{Statistical Analysis}

Fisher's exact test of independence was used to assess differences in categorical variable proportions. Incidence rates of secondary de novo CMs were modeled as Poisson distributions and exact Poisson testing was used to evaluate differences in rate parameters. Kaplan-Meier methods were used to elucidate significant relationships between patient- and treatment-related covariates and outcome metrics (e.g., progression-free survival, incidence of de novo lesions, etc.). Logistic regression was used to evaluate associations between CM location and resection extent. Univariable and multivariable Cox proportional hazards regression was used to evaluate risk of developing de novo lesions and risk of future symptomatic lesions requiring resection. Covariates were included in the multivariable model if the associated univariable $p$ value was $<0.1$. The recurrence rate between patients receiving GTR and STR was compared using Kaplan-Meier methods. All statistical testing was 2-sided, and significance was evaluated based on an $\alpha$ statistic of 0.05 . All statistical and graphical analyses were conducted using R (version 3.5.2, The R Project for Statistical Computing) and GraphPad Prism 8 (GraphPad Software).

\section{Results}

\section{Patient Characteristics}

Patient characteristics are detailed in Table 1 and patient examples in Fig. 2. The study group included 32 male and 21 female patients with a ratio of approximately 1.5:1. The mean age at first surgery was 10.5 years with a range of 0.3-18 years. Acute hemorrhage based on imaging was present in $64 \%$ of patients on presentation. The most common presenting symptom was seizure (45\%), followed by neurological deficit (34\%), severe headache or mild headache with a known family history $(8 \%)$, and somnolence (6\%). Eight percent of cases were found incidentally. Single CMs were found in 57\% (30/53) of patients on initial MRI, whereas $42 \%$ (22/53) of patients had multiple CMs. One patient died prior to MRI; thus, the number of CMs was unknown. Thirteen (25\%) of the 53 patients had a known family history of CMs. The most common location of symptomatic lesions was cortical (58\%), followed by brainstem (18\%), basal ganglia/thalamus (9\%), cerebellar $(8 \%)$, lesions extending into more than one location $(5 \%)$, and cervicomedullary (1\%).

The 53 patients underwent a total of 74 operations for resection of CMs at our institution. Two patients had histories of CM resections at a younger age at other institutions. Two patients who required multiple operations had arteriovenous malformations resected in addition to their CMs. Forty-three patients (81\%) underwent a single operation for resection of a $\mathrm{CM} ; 10$ patients $(19 \%)$ underwent more than one operation (Table 1).

In general, the indications for surgery were if the $\mathrm{CM}$ was thought to be causing symptoms such as seizures or neurological deficits. Eight patients presenting with head- 


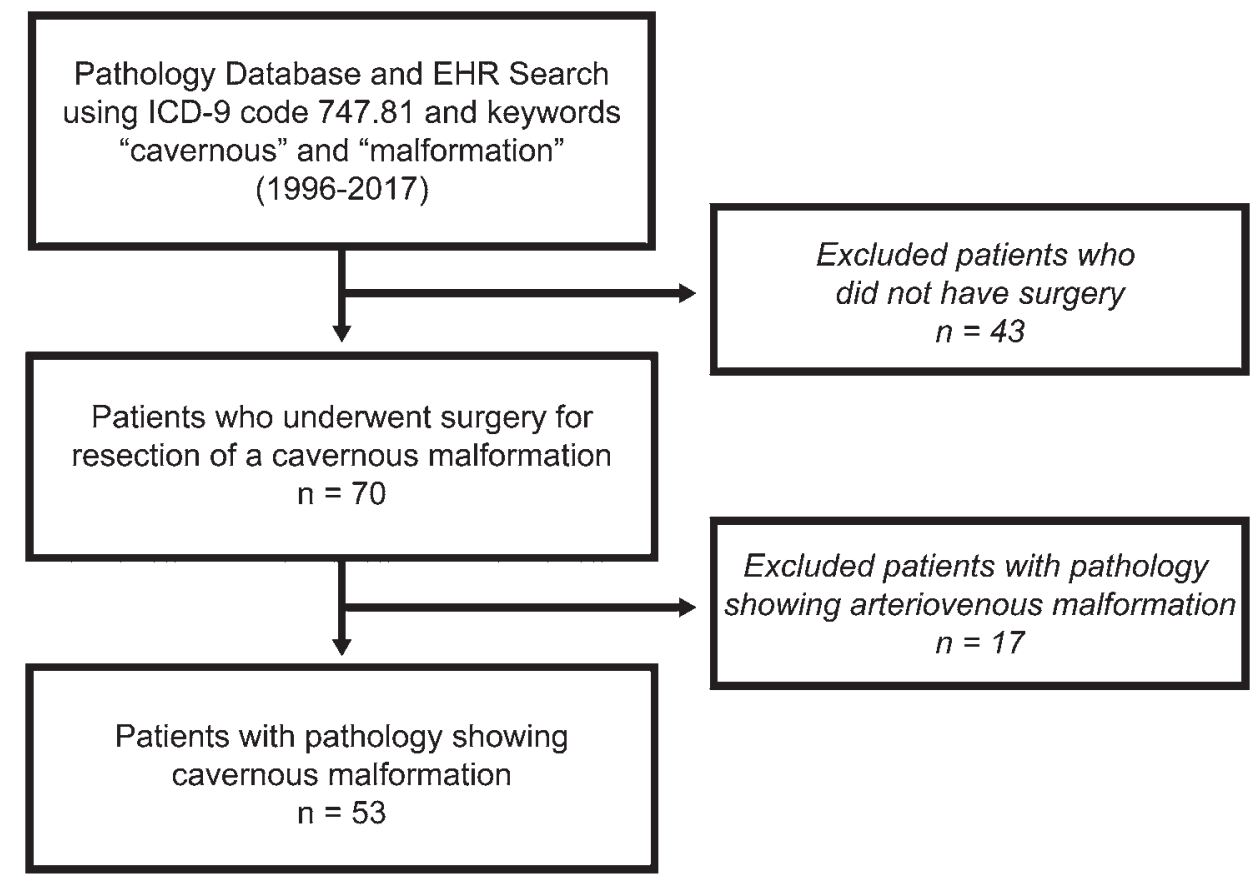

FIG. 1. CONSORT diagram showing CM inclusion and exclusion criteria. EHR = electronic health record.

ache or who had an incidentally found CM underwent surgery. Two of these patients had acute hemorrhage on imaging. Of the 6 without acute hemorrhage, 4 patients had multiple CMs and 2 had a single CM. For these patients the surgeon was concerned about the size or enlarging size of the CM. For patients with seizures or neurological deficits, the CM that showed acute hemorrhage or was thought to be related to these findings was resected in cases of multiple lesions. For patients with medically intractable seizures, the goal of surgery was to resect both the CM and the hemosiderin in the surrounding white matter.

Twenty resected CMs (38\% of initial resections) had an associated DVA that was preserved (Table 1). An additional 3 patients had CMs elsewhere associated with a DVA. Patients presenting with multiple CMs on initial MRI were less likely to have a DVA associated with the resected CM. Excluding 2 patients in whom the presence of a DVA was uncertain, 5 (23\%) of the 22 patients presenting with multiple CMs on initial MRI had an associated DVA compared to $15(54 \%)$ of the 28 patients presenting with single CM on initial MRI $(\mathrm{p}=0.042)$. Presence of DVA was unknown for 3 patients in total. Patients who had a DVA on initial MRI were no more likely to present with acute hemorrhage (15 of 22 patients, 68\%) compared to patients who did not have a DVA on initial MRI (17 of 26 patients, $65 \%$; $\mathrm{p}=1.000)$.

\section{Recurrence Rate of Resected CMs}

Thirty-six (68\%) of the 53 initial CMs were completely resected both by surgeon report and postoperative imaging. Thirteen patients (25\%) showed STR and $4(8 \%)$ did not have follow-up imaging. Brainstem lesion location was associated with a higher likelihood of STR (OR 4.41, 95\% CI 1.19-17.16). Neither cerebellar (OR 1.89, 95\% CI
0.24-11.43) nor basal ganglia/thalamic (OR 1.26, 95\% CI 0.06-11.27) location was associated with extent of resection. Low numbers limited analysis for mixed location and spinal cord CMs. Compared to $81 \%$ of cortical CMs, only $46 \%$ of brainstem CMs underwent GTR.

Including subsequent symptomatic lesions requiring resection after the initial procedure, a larger fraction of subtotally resected CMs demonstrated radiographic progression at 1 year compared to radiographic recurrence at 1 year in CMs that were completely resected (4/21 [STR; $19 \%$ ] vs $1 / 47$ [GTR; 2.1\%], $p=0.029$; Fig. 3A). In the total follow-up period, $12 \mathrm{CMs}$ recurred or progressed with a highly variable time to radiographic recurrence or progression (average 5.1 years, SD 5.2).

The annual rate of recurrence was $0.7 \%$ after GTR (2 recurrences in 290.0 lesion-years of follow-up) compared to an annual rate of progression of $11.9 \%$ after STR (10 progressions in 84.3 lesion-years of follow-up) $(\mathrm{p}<0.001)$. Recurrence-free survival was also greater for gross-totally resected lesions compared to progression-free survival in subtotally resected lesions (hazard ratio [HR] 23.51, 95\% CI 6.31-87.56; Fig. 3B). Only 7 (58\%) of the recurrent or progressive lesions required re-resection. In general, reresection was done for recurrence of symptoms and/or progressively enlarging residual CMs. Ten recurrent or progressive lesions (83\%) were in patients presenting with multiple CMs and 2 were in patients with a single CM. Sixty-one CMs did not recur or progress (31 in patients with multiple CMs and 30 in patients with single CMs).

\section{De Novo CMs}

Next, we were interested in whether the number of CMs on initial MRI was predictive of the number of CMs on subsequent MRI. We noted 2 distinct populations of 
TABLE 1. Characteristics in 53 patients with CMs

\begin{tabular}{|c|c|c|}
\hline Characteristic & No. & $\%$ \\
\hline No. of patients & 53 & \\
\hline No. of resected CMs & 74 & \\
\hline Mean age at 1st op, yrs & 10.5 & Range $0.3-18$ \\
\hline \multicolumn{3}{|l|}{ Sex } \\
\hline Male & 32 & 60 \\
\hline Female & 21 & 40 \\
\hline \multicolumn{3}{|l|}{ Ethnicity } \\
\hline Caucasian & 29 & 55 \\
\hline Hispanic & 8 & 15 \\
\hline Non-Hispanic & 7 & 13 \\
\hline Asian & 6 & 11 \\
\hline Unknown & 3 & 6 \\
\hline \multicolumn{3}{|l|}{ Symptoms at presentation } \\
\hline Seizure & 24 & 45 \\
\hline Neurological deficit & 18 & 34 \\
\hline Severe headache & 4 & 8 \\
\hline Somnolence & 3 & 6 \\
\hline Incidental finding & 4 & 8 \\
\hline \multicolumn{3}{|l|}{ Associations } \\
\hline DVA w/ resected CM & 20 & 38 \\
\hline DVA w/ distant CM & 3 & 6 \\
\hline AVM & 2 & 4 \\
\hline \multicolumn{3}{|c|}{ Number of lesions on initial MRI } \\
\hline Single & 30 & 57 \\
\hline Multiple & 22 & 42 \\
\hline Unknown & 1 & 2 \\
\hline \multicolumn{3}{|l|}{ Acute hemorrhage at $D x$} \\
\hline Yes & 34 & 64 \\
\hline No & 19 & 36 \\
\hline History of radiation & 1 & 2 \\
\hline Known family history of $\mathrm{CM}$ & 13 & 25 \\
\hline \multicolumn{3}{|l|}{ Lesion location $(n=74)$} \\
\hline Supratentorial cortical & 43 & 58 \\
\hline Brainstem & 13 & 18 \\
\hline Basal ganglia/thalamus & 7 & 9 \\
\hline Cerebellar & 6 & 8 \\
\hline Mixed & 4 & 5 \\
\hline Spinal cord & 1 & 1 \\
\hline \multicolumn{3}{|l|}{ No. of ops } \\
\hline 1 & 43 & 81 \\
\hline 2 & 4 & 8 \\
\hline 3 & 3 & 6 \\
\hline 4 & 2 & 4 \\
\hline 5 & 0 & 0 \\
\hline 6 & 1 & 2 \\
\hline \multicolumn{3}{|l|}{ Follow-up } \\
\hline Mean (person-yrs) & 6.8 & Range $0.2-22$ \\
\hline Total person-yrs & 375 & NA \\
\hline Total lesion-yrs & 505 & NA \\
\hline
\end{tabular}

$\mathrm{AVM}=$ arteriovenous malformation; $\mathrm{Dx}=$ diagnosis; $\mathrm{NA}=$ not applicable. patients. One population presented with a single symptomatic CM $(\mathrm{n}=30,58 \%)$ and only $1(3.3 \%)$ of the 30 patients went on to develop further CMs. The only patient who presented with a single $\mathrm{CM}$ and was found to have more than one lesion on subsequent MRI had a history of cranial radiation. The second population presented with multiple CMs on initial MRI $(n=22,42 \%)$. In this population, $15(71 \%)$ of the 21 patients with subsequent imaging were found to have de novo CMs not apparent on initial MRI, which was significantly more frequent than in patients presenting with only a single symptomatic $\mathrm{CM}(\mathrm{p}$ $<0.001$ ). Incidence rates of additional de novo CMs were 6.6 and 78.8 lesions per 1000 person-years of follow-up for patients presenting with single and multiple malformations, respectively, on initial MRI ( $p=0.002)$.

On univariable analysis, patients presenting with preoperative symptoms other than seizure and neurological deficits (HR 5.38, 95\% CI 1.02-28.4), patients with multiple CMs at first imaging (HR 14.88, 95\% CI 1.94-114.1; Fig. 3C), and patients receiving an STR initially (HR 4.88, 95\% CI 1.61-14.8) were more likely to develop de novo lesions at a later time point. After accounting for included covariates, only the presence of multiple lesions on initial imaging was independently prognostic for the development of subsequent de novo CMs (adjusted hazard ratio [aHR] 8.61, 95\% CI 1.01-73.30; Supplemental Table 1).

\section{Characteristics of Symptomatic CMs}

In total, the 53 patients underwent 74 operations for resection of CMs. Two (6.9\%) of the 29 patients who presented with a single $\mathrm{CM}$ had additional resections and 8 $(38.1 \%)$ of the 21 patients who presented with more than one $\mathrm{CM}$ had additional resections $(\mathrm{p}=0.011)$. One patient from each group did not have follow-up. One patient who required an emergency craniotomy for evacuation of an acute hemorrhage did not have an initial MRI study and died on postoperative day 4 from intracranial hypertension.

Overall, for subsequent surgeries, $38.1 \%(n=8)$ were for other CMs present on initial MRI, $28.6 \%(n=6)$ were for residual lesions, $23.8 \%(\mathrm{n}=5)$ were for de novo lesions not present on initial MRI, and 9.5\% $(n=2)$ were for recurrent lesions (Fig. 4A). Of the 2 patients requiring surgery for a recurrent lesion, one had a single $\mathrm{CM}$ on initial imaging and the other had multiple CMs. On unadjusted analysis, having multiple CMs at diagnosis was correlated with more frequent secondary surgeries (HR 5.26, 95\% CI 1.11-24.86; Fig. 4B and Table 2). Being of Asian ethnicity (HR 5.84, 95\% CI 1.28-26.57; Table 2) was also associated with an increased risk of additional resections. When only considering patients undergoing re-resection for recurrent or residual lesions (not considering de novo lesions and lesions present on initial MRI), achieving GTR was associated with reduced incidence of repeat surgeries (HR 0.21, 95\% CI 0.04-0.99; Fig. 4C). After adjusting for patient- and lesion-specific characteristics in a multivariable framework, the presence of acute radiographic hemorrhage at initial diagnosis (aHR 27.84, 95\% CI 1.02760.55 ) and having multiple lesions at presentation (aHR $8.43,95 \%$ CI $1.09-65.41$ ) were independently associated with the development of subsequent symptomatic lesions requiring resection (Fig. $4 \mathrm{D}$, Table 2 ). 

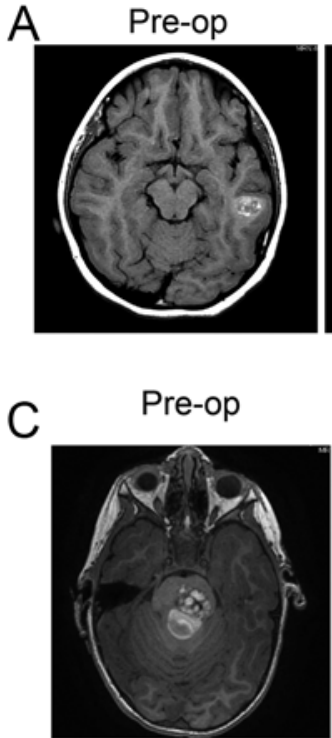

$t=0$

Existing $\mathrm{CM}$

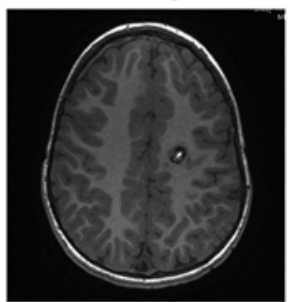

$t=0$

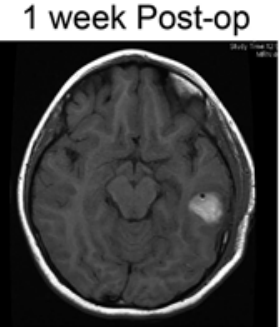

1 week Post-op

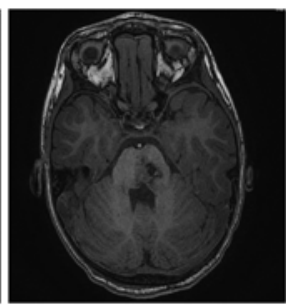

$t=1$ wk

de novo $\mathrm{CM}$

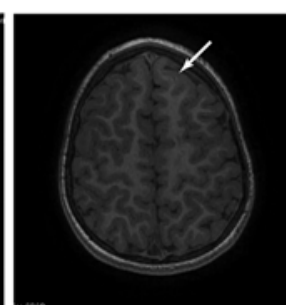

$t=2 \mathrm{yrs}$

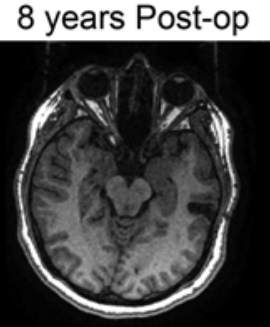

2 years Post-op

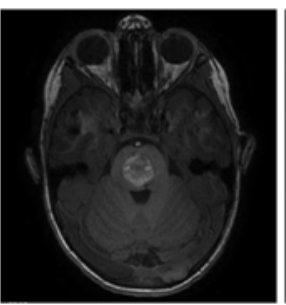

$t=2$ yrs

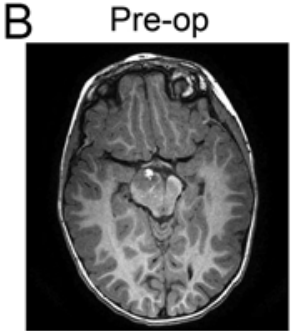

2 wks Post-op 2nd resection

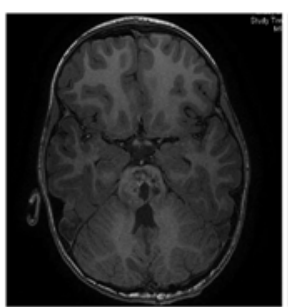

$t=2$ yrs, $2 w k s$
6 weeks Post-op
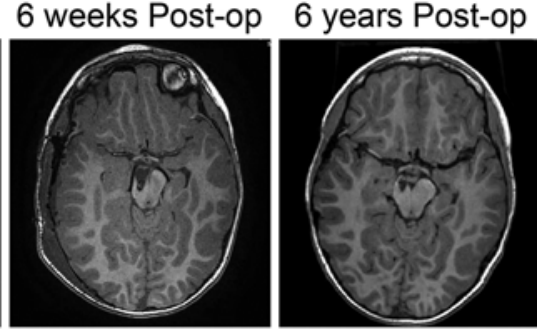

3 yrs Post-op 3rd resection

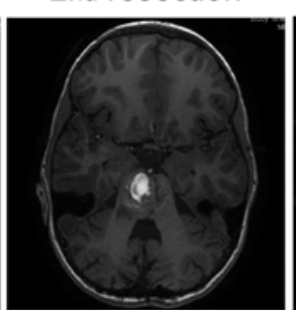

$t=4$ yrs

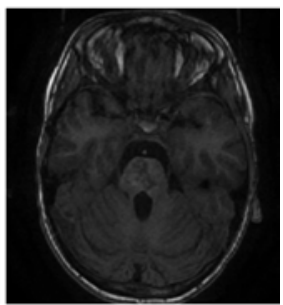

$t=7$ yrs

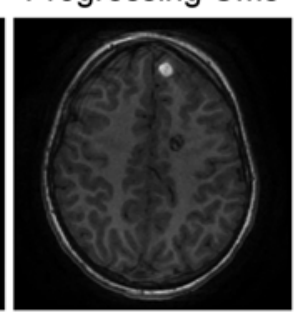

$t=7$ yrs

FIG. 2. Illustrative cases investigated with MRI. A: An 8-year-old girl presented with aphasia and altered mental status and was found to have a left temporal hemorrhage (left). She underwent GTR of her single CM (center), which has not recurred after 8 years (right). B: A 3-year-old boy presented with headache, emesis, third nerve palsy, and left-sided weakness. MRI demonstrated a right upper ventral midbrain hemorrhage (left). He underwent GTR of his single CM (center), which has not recurred after 6 years (right). C: A 3-year-old boy with a family history of CMs presented with right-sided weakness and right facial palsy. MRI demonstrated a pontine diencephalic CM with hemorrhage (upper row, first image) and a second supratentorial CM without acute hemorrhage (lower left). He underwent a GTR (upper row, second image). Two years later he presented with a severe headache and nausea and was found to have hemorrhaged into the area of his previously resected pontomedullary CM (upper row, third image). His left frontal CM was slightly enlarged without evidence of acute hemorrhage, and a de novo left frontal lobe CM was also identified (lower center, arrow). The subtotally resected CM (upper row, fourth image) progressed (upper row, fifth image) and the patient returned with a sixth nerve palsy and left-sided dysmetria. The patient underwent a GTR, with no residual or recurrent CM noted on imaging 3 years later (upper row, sixth image). The patient remained asymptomatic from the 2 frontal CMs, which have enlarged over time (lower right).

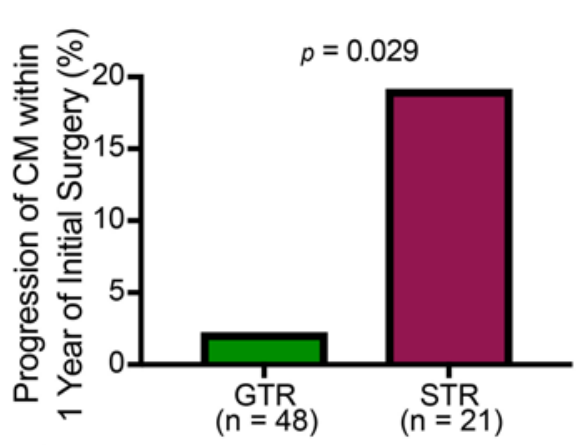

A

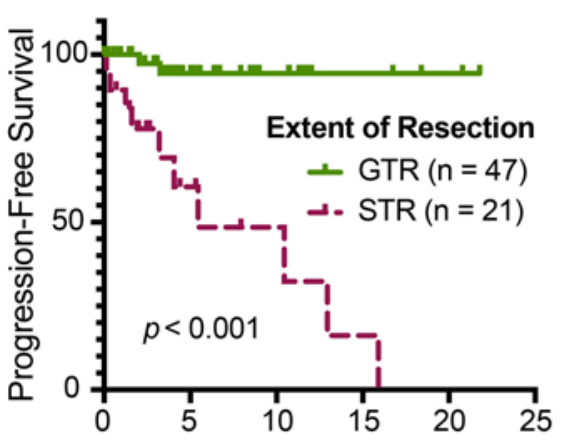

B Time Following Surgery (Years)

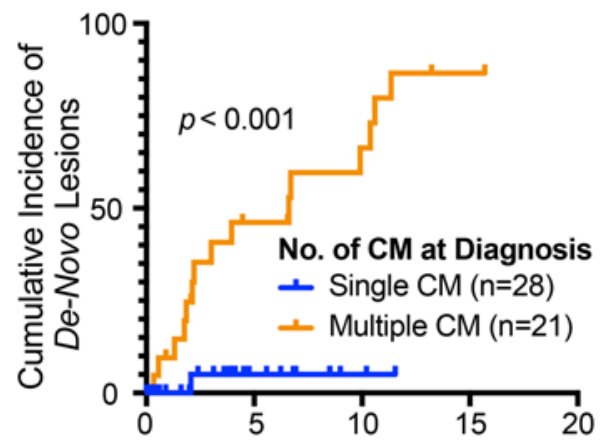

C Time Following Surgery (Years)

FIG. 3. Lesion recurrence and de novo CMs. A: Patients undergoing GTR demonstrate lower 1-year lesion recurrence rates than patients undergoing STR. B: Totally resected CMs have a significantly lower recurrence rate than subtotally resected lesions. C: The presence of multiple CMs at diagnosis is associated with an increased incidence of future de novo CMs. 
A

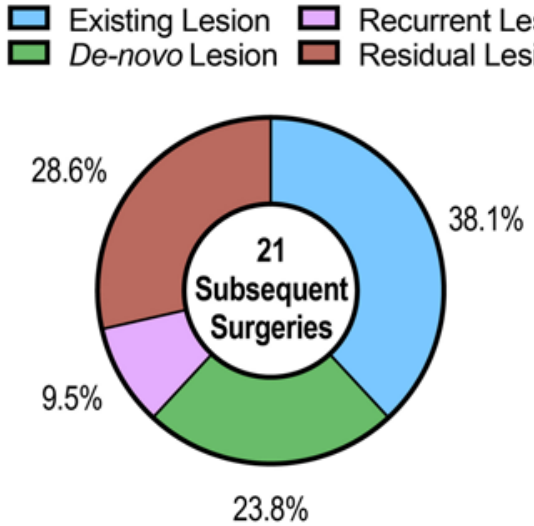

C

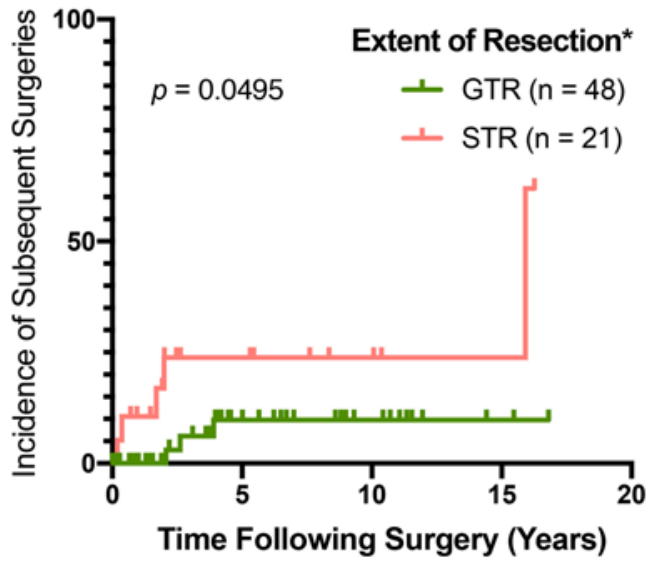

B

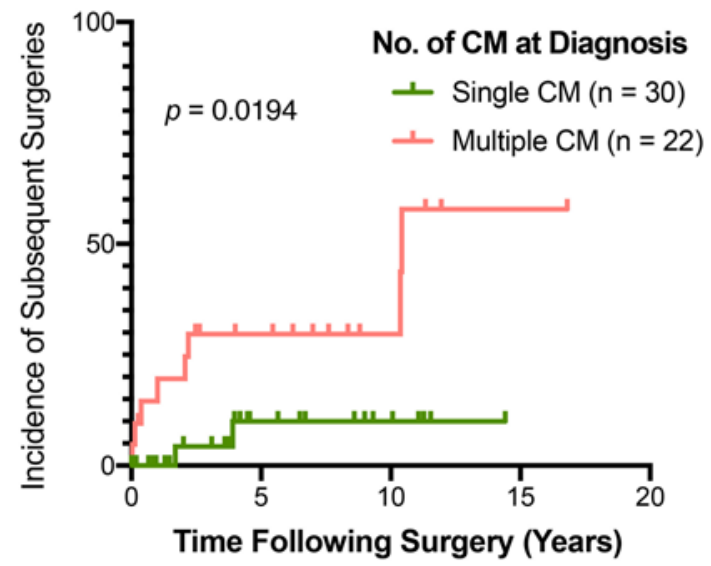

D
Multivariable Cox Model

(Time to Subsequent Surgery)

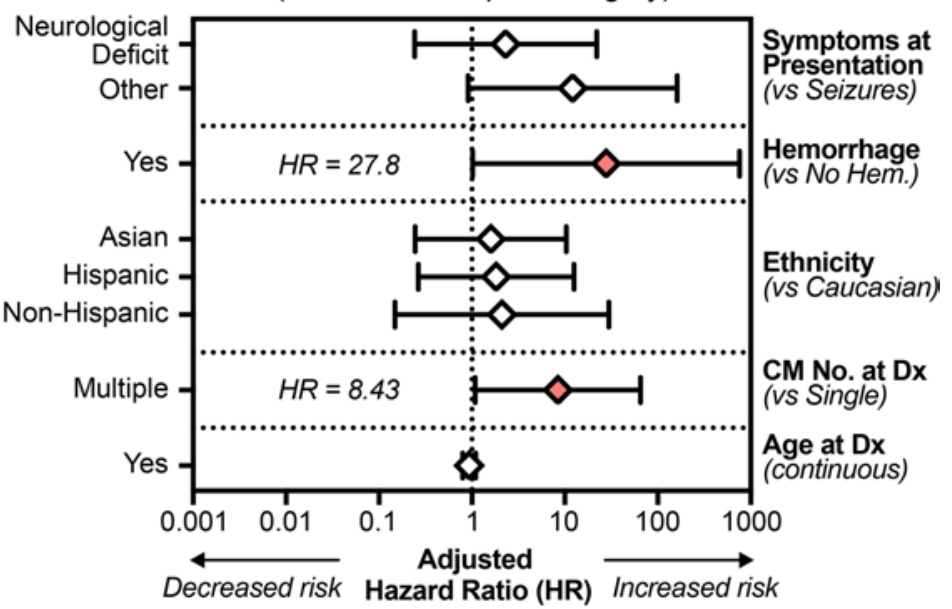

FIG. 4. Analysis of subsequent surgeries. A: Reasons for subsequent surgeries in pediatric patients with resected CMs. B: The incidence of subsequent surgeries is associated with the number of CMs present at the first diagnosis. C: GTR is associated with lower rates of subsequent surgeries for previously resected lesions compared to STR. Asterisk denotes the fact that extent of resection only includes surgeries for residual or recurrent lesions. D: Acute hemorrhage at diagnosis and the presence of multiple CMs are independently associated with an increased risk of subsequent surgeries.

\section{Heuristic Classification of Risk for Subsequent Symptomatic CMs}

Patients were classified heuristically as being low risk (presenting initially with neither of the risk factors [acute hemorrhage or multiple lesions]), medium risk (presenting with one risk factor), or high risk (presenting with both risk factors; Fig. 5). None of the patients in the low-risk group developed additional symptomatic CMs requiring resection (50.2 total person-years of follow-up after initial resection). In patients with medium risk, a total of 4 subsequent surgeries were required for new symptomatic lesions, indicating an annual rate of $2.0 \%$ per patient (198.2 total person-years of follow-up after initial resection). High-risk patients received 17 additional surgeries for symptomatic lesions after the first resection, corresponding to an annual rate of $13.5 \%$ per person (126.1 total person-years of follow-up after initial resection). High-risk patients developed symptomatic lesions requiring resection at a significantly faster rate than both the low-risk group $(\mathrm{p}=0.005)$ and the medium-risk group $(\mathrm{p}<0.001)$.
The recurrence rate also differed between patients in different risk categories. No lesions recurred in low-risk patients in 22.9 lesion-years of follow-up ( $0.0 \%$ annual risk). Four resected CMs recurred in medium-risk patients in 167.3 lesion-years (2.4\% annual risk), whereas 8 resected CMs recurred in high-risk patients in 184.1 lesion-years (4.3\% annual risk). Preoperative symptoms improved in all low-risk patients following surgery, whereas $86 \%$ of medium-risk patients and $75 \%$ of high-risk patients experienced symptomatic relief after surgery.

\section{Postoperative Patient Monitoring}

Postoperative and follow-up MRI study timing was aggregated to identify patterns of care following surgery. Patterns of imaging follow-up were heterogeneous, even when grouped by the number of surgeries received. In patients with at least 30 days of follow-up, the median number of MRI studies per year did not differ between patients eventually receiving multiple surgeries and those receiving a single surgery $(1.002$ vs $1.154, p=0.3435)$. Stratifica- 
TABLE 2. Univariable and multivariable Cox proportional hazards model (secondary symptomatic lesions) in patients with CMs

\begin{tabular}{|c|c|c|c|c|c|c|}
\hline \multirow[b]{2}{*}{ Covariate } & \multicolumn{3}{|c|}{ Univariable } & \multicolumn{3}{|c|}{ Multivariable } \\
\hline & $\mathrm{HR}$ & $95 \% \mathrm{Cl}$ & p Value & $\mathrm{HR}$ & $95 \% \mathrm{Cl}$ & $\mathrm{p}$ Value \\
\hline Age at initial op (continuous) & 0.909 & 0.815 to 1.015 & 0.090 & 0.936 & 0.794 to 1.103 & 0.466 \\
\hline Year of Dx (continuous) & 0.910 & 0.797 to 1.038 & 0.161 & & & \\
\hline \multicolumn{7}{|l|}{ Sex } \\
\hline Male (ref) & 1 & NA & NA & NA & NA & NA \\
\hline Female & 0.693 & 0.211 to 3.187 & 0.774 & & & \\
\hline \multicolumn{7}{|l|}{ Ethnicity } \\
\hline Caucasian (ref) & 1 & NA & NA & 1 & NA & NA \\
\hline Asian & 5.838 & 1.283 to 26.57 & 0.023 & 1.599 & 0.245 to 10.42 & 0.624 \\
\hline Hispanic (nonwhite) & 1.423 & 0.231 to 8.776 & 0.704 & 1.822 & 0.265 to 12.53 & 0.542 \\
\hline Non-Hispanic (nonwhite) & 1.123 & 0.114 to 11.11 & 0.921 & 2.099 & 0.148 to 29.71 & 0.583 \\
\hline \multicolumn{7}{|l|}{ Preop symptoms } \\
\hline Seizure (ref) & 1 & NA & NA & 1 & NA & NA \\
\hline Neurological deficits & 7.866 & 0.945 to 65.46 & 0.056 & 2.315 & 0.242 to 22.15 & 0.466 \\
\hline Other & 7.653 & 0.793 to 73.86 & 0.079 & 12.14 & 0.912 to 161.5 & 0.059 \\
\hline \multicolumn{7}{|l|}{ Lesion location } \\
\hline Cortical (ref) & 1 & NA & NA & NA & NA & NA \\
\hline Noncortical & 1.793 & 0.518 to 6.213 & 0.357 & & & \\
\hline \multicolumn{7}{|l|}{ DVA present } \\
\hline No (ref) & 1 & NA & NA & NA & NA & NA \\
\hline Yes & 2.474 & 0.689 to 8.881 & 0.165 & & & \\
\hline \multicolumn{7}{|l|}{ Acute hemorrhage at Dx } \\
\hline No (ref) & 1 & NA & NA & NA & NA & NA \\
\hline Yes & 6.887 & 0.827 to 57.38 & 0.074 & 27.84 & 1.019 to 760.6 & 0.049 \\
\hline \multicolumn{7}{|l|}{ Number of CMs at Dx } \\
\hline Single (ref) & 1 & NA & NA & 1 & NA & NA \\
\hline Multiple & 5.258 & 1.112 to 24.86 & 0.036 & 8.430 & 1.087 to 65.41 & 0.041 \\
\hline \multicolumn{7}{|l|}{ Resection extent } \\
\hline GTR (ref) & 1 & NA & NA & NA & NA & NA \\
\hline STR & 2.855 & 0.756 to 10.79 & 0.122 & & & \\
\hline
\end{tabular}

Boldface type indicates statistical significance.

tion based on risk strata assigned by our heuristic model also demonstrated no difference in radiographic follow-up (Kruskal-Wallis, $\mathrm{p}=0.402$ ), and high-risk patients were not imaged more frequently than low-risk (median 0.930 MRI studies/year vs $1.183, \mathrm{p}=0.384$ ) or medium-risk (median 0.984 MRI studies/year vs $1.183, \mathrm{p}=0.208$ ) patients.

\section{Postsurgical Complications}

In total there were 6 major complications with a total complication rate of $8.6 \%$. The most serious was a death. This was in a 13-year-old girl who presented with confusion, headache, emesis, and a seizure and was found to have a large acute left frontal lobe hemorrhage with associated mass effect, midline shift, and hydrocephalus on CT scan. She was brought to the operating room for emergency evacuation of hemorrhage and external ventricular drain placement. She died on postoperative day 4 from cerebral edema with severe intracranial hypertension, thalamic in- farct, and electrolyte abnormalities. One patient had a right thalamic/internal capsule venous infarct leading to left-sided weakness and hemianopia, which gradually improved to a near-normal neurological examination over the course of 9 months. Another patient had a deep wound infection requiring removal of the bone flap. Two patients had CSF leaks requiring surgical repair, with one eventually requiring a ventriculoperitoneal shunt. One patient had a pseudomeningocele requiring a lumbar drain.

\section{Discussion}

CMs are a common cerebrovascular anomaly in children, and this is the first large case series describing outcomes following resection. We found an annual recurrence rate following complete resection of $0.7 \%$. We had a large proportion of children presenting with multiple CMs on initial imaging, allowing us to identify a higher rate of de novo lesions in this population. GTR of CMs did reduce 


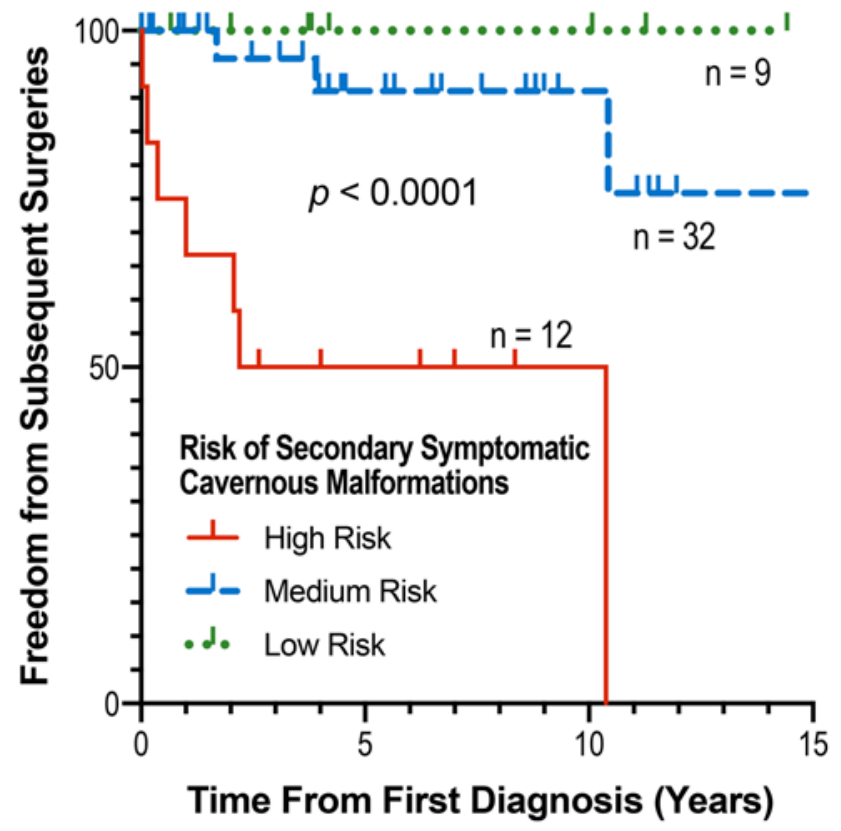

FIG. 5. Heuristic risk model for likelihood of subsequent symptomatic $\mathrm{CMs}$ requiring resection. Patients presenting with both acute hemorrhage and multiple CMs at diagnosis are at highest risk for requiring additional surgeries, whereas patients with neither risk factor are at lowest risk.

the incidence of subsequent surgeries for residual or recurrent lesions compared to STR. However, since the majority of subsequent surgeries were for other CMs on initial MRI and de novo lesions rather than for residual/recurrent lesions, GTR did not reduce the incidence of subsequent surgeries overall on a patient level. Multivariable analysis identified acute hemorrhage and the presence of multiple $\mathrm{CMs}$ at initial presentation as independent prognostic factors for increased risk of subsequent surgeries for symptomatic CMs. We present a heuristic risk model that stratifies patients into high-, medium-, and low-risk groups (Fig. 5) that could allow for the development of risk-adapted clinical and radiological monitoring strategies (Fig. 6).

Although the natural history of pediatric CMs has been studied ${ }^{5}$ outcomes following resection of symptomatic CMs have been less explored. Postoperative management following resection of a symptomatic CM is unstandardized, ${ }^{6}$ even at our institution, in part because the risk of future events has not been investigated. To propose guidelines to standardize postoperative care and to counsel patients on the future risk of requiring additional surgery, we analyzed the factors that drove reoperations in pediatric patients who underwent resection of at least one symptomatic CM.

Radiographic progression, defined as acute or subacute hemorrhage in the location of a previously resected CM, was associated with incomplete resection of the initial lesion. Prior studies have also demonstrated the association of STR with future hemorrhagic events. ${ }^{7}$ However, the association between subsequent neurological symptoms attributable to CM regrowth that requires surgical intervention and radiographic findings is not well understood. In our study we aimed to assess radiographic endpoints both independently and in the context of subsequent surgical care. As expected, repeat surgeries for residual or recurrent lesions were more frequent in patients receiving STR compared to GTR. However, only a minority of second surgeries were prompted by radiographic evidence of recurrent or residual lesion on routine imaging, with the majority of subsequent surgeries directed at newly symptomatic lesions that had been previously present or had emerged de novo.

DVAs are abnormal complexes of venous return demonstrated to coincide with CMs in up to $33 \%$ of patients. ${ }^{8}$ Unlike patients with familial CMs, who often present with multiple lesions and whose CMs are associated with heritable mutations in $C C M 1, C C M 2$, and $C C M 3$, patients with sporadic CMs more frequently present with DVAs, with prior studies suggesting that preexistence of DVAs potentiates development of subsequent vascular malformations. ${ }^{9-11}$ The impact of an associated DVA on CM presentation is unclear. Gross et al. found that CMs associated with DVAs were more likely to present with hemorrhage, defined as the presence of acute bleeding visualized on imaging and accompanied by neurological symptoms; ${ }^{5}$ however, we did not find that CMs associated with DVAs were more likely to present with hemorrhage. In our cohort, patients presenting with multiple CMs were less likely to have an associated DVA compared to patients presenting with a single $\mathrm{CM}$.

Because our study was aimed at elucidating the clinical course that pediatric patients experience following an initial surgery for a symptomatic CM, we sought to understand the factors affecting the development of subsequent symptomatic CMs requiring further intervention. Only acute hemorrhage at first diagnosis and the presence of multiple lesions were independently associated with increased risk of requiring subsequent surgeries. Based on this, we defined a heuristic risk classifier stratifying patients based on the likelihood of future symptomatic lesions, regardless of etiology, that require surgical intervention (Fig. 5). We found that no patients in the low-risk group required additional surgeries and $50 \%$ of patients in the high-risk group required additional surgery by 2.5 years after initial resection. Using this model we propose treatment guidelines based on risk of an individual patient to require further intervention (Fig. 6). In general, we propose that patients in the low-risk group obtain imaging immediately postoperatively, at 1 month, and at 1 year. If imaging shows stable results they do not necessarily require further routine imaging. In contrast, patients in the highrisk group should have imaging immediately postoperatively, at 1 month, at 6 months, and then yearly given the increased risk of developing further symptomatic lesions.

\section{Study Limitations}

Limitations of the study include the unmeasured influence of variables not collected, the absence of an independently treated validation cohort, and the retrospective nature of our study. Although emergence of symptoms attributable to the CM was an indication for surgical intervention, it is possible that confounding factors of nonbiological origin influence the decision to resect a $\mathrm{CM}$. 


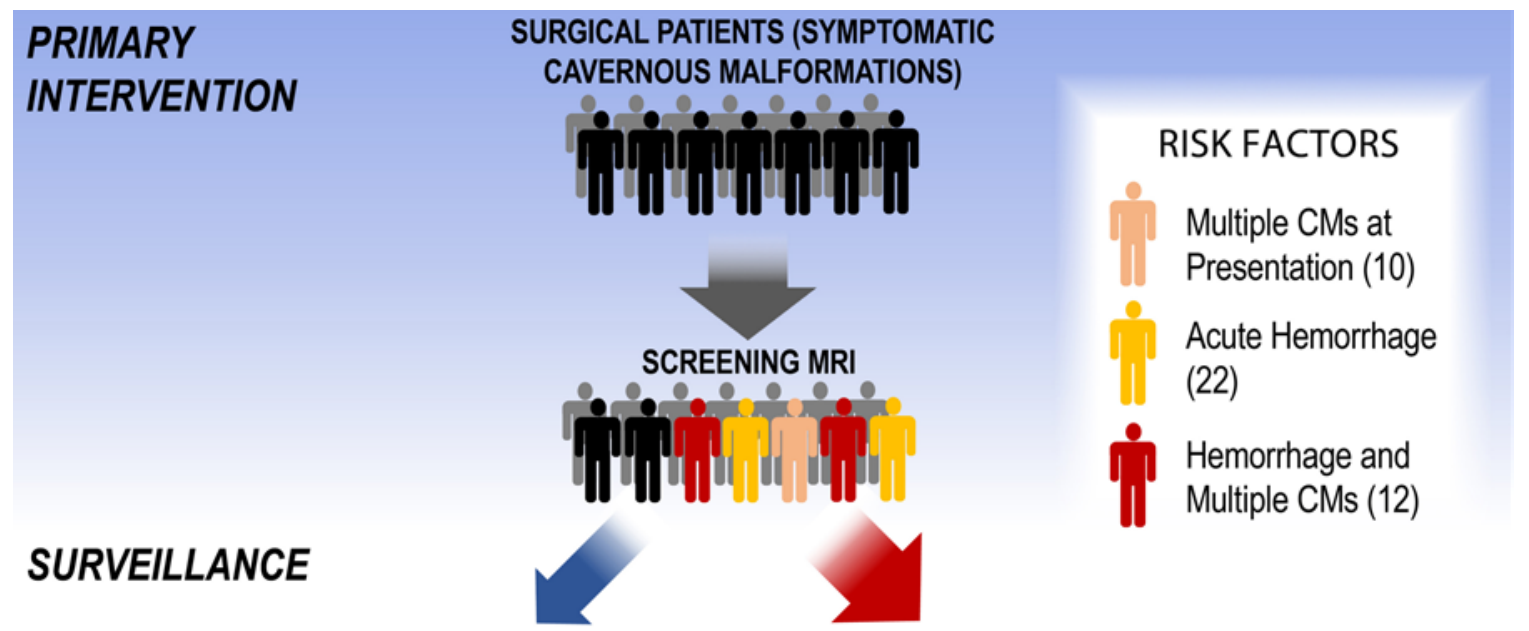

Low Risk for Additional Symptomatic Lesions
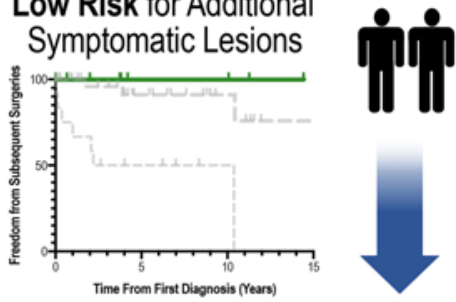

Surveillance MRI

(immediate post-op, 1 month, 1 year)
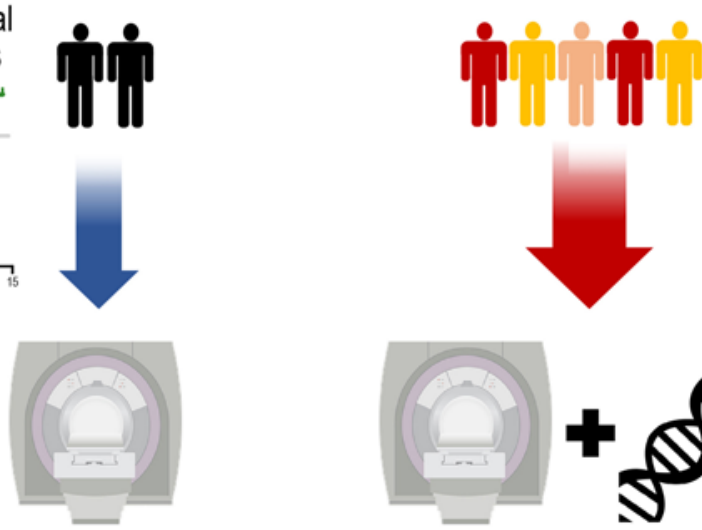

Medium or High Risk for Additional Symptomatic Lesions

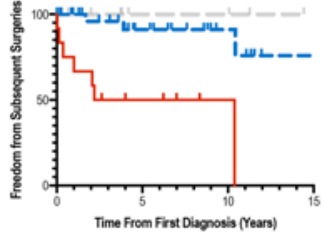

Surveillance MRI

(immediate post-op, 1 month, 6 months, yearly)

Genetic Testing

\section{SECONDARY INTERVENTION}

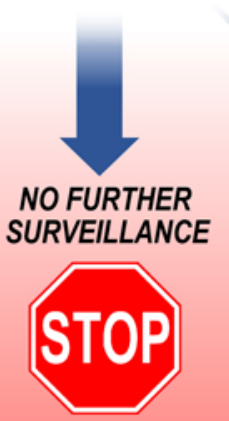

NO LESIONS

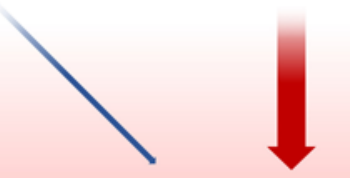

SURGERY

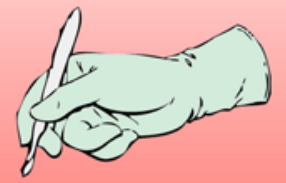

SYMPTOMATIC LESION

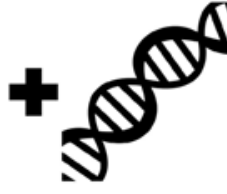

FIG. 6. Proposed treatment guideline. Proposed guidelines for systematically monitoring patients based on the risk of subsequent symptomatic CMs based on initial imaging. Postoperative patients are considered low risk (black figures, left) for requiring subsequent surgery if they present with a single CM on initial MRI and without acute hemorrhage. Postoperative patients are considered medium risk if they present with either multiple CMs (tan figures, right) or acute hemorrhage (yellow figures, right). Postoperative patients are considered high risk for requiring additional surgeries in the future if they present with multiple CMs and acute hemorrhage on initial imaging (red figures, right). In our series, none of the patients in the low-risk group required additional surgeries. Thirty-four patients presented with acute hemorrhage (22 medium-risk and 12 high-risk individuals) and 22 patients with multiple CMs (10 medium-risk and 12 high-risk individuals). Although at this time genetic testing does not directly affect treatment decisions for CMs, genetic testing may influence the frequency of imaging and screening of family members. A specific regimen for MRI surveillance is noted but is still under investigation prospectively. † Asymptomatic lesions include de novo, recurrent, and residual lesions.

Additionally, due to the relatively small size of our investigational cohort, we did not include a separate validation cohort for the proposed heuristic risk model. Ideally, inclusion of an independently treated set of patients managed by a distinct set of neurosurgeons would demonstrate ro- bustness of the findings presented in this study. To address these concerns, future investigations into the causal factors determining the emergence of symptomatic CMs should be conducted in a prospective, randomized setting.

Additionally, our cohort consisted of patients who had 
already undergone resection of pathologically proven CMs. We do not address the natural history of CMs in patients who present with incidentally found single or multiple lesions to analyze which lesions become symptomatic.

\section{Conclusions}

Pediatric patients presenting with CMs requiring surgical intervention have differential future risks of requiring subsequent surgery based on the number of lesions present on initial imaging and the presence of acute hemorrhage. Longitudinal follow-up should be risk adapted for increased surveillance uniformity and improved clinical decision-making.

\section{References}

1. Awad IA, Polster SP. Cavernous angiomas: deconstructing a neurosurgical disease. J Neurosurg. 2019;131(1):1-13.

2. Batra S, Lin D, Recinos PF, et al. Cavernous malformations: natural history, diagnosis and treatment. Nat Rev Neurol. 2009;5(12):659-670.

3. Kosnik-Infinger L, Carroll C, Greiner H, et al. Management of cerebral cavernous malformations in the pediatric population: a literature review and case illustrations. J Neurosurg Sci. 2015;59(3):283-294.

4. Mottolese C, Hermier M, Stan H, et al. Central nervous system cavernomas in the pediatric age group. Neurosurg Rev. 2001;24(2-3):55-73.

5. Gross BA, Du R, Orbach DB, et al. The natural history of cerebral cavernous malformations in children. J Neurosurg Pediatr. 2016;17(2):123-128.

6. Akers A, Al-Shahi Salman R, Awad IA, et al. Synopsis of Guidelines for the Clinical Management of Cerebral Cavernous Malformations: consensus recommendations based on systematic literature review by the Angioma Alliance Scientific Advisory Board Clinical Experts Panel. Neurosurgery. 2017;80(5):665-680.

7. Li D, Hao SY, Tang J, et al. Surgical management of pediatric brainstem cavernous malformations. J Neurosurg Pediatr. 2014;13(5):484-502.
8. Töpper R, Jürgens E, Reul J, Thron A. Clinical significance of intracranial developmental venous anomalies. J Neurol Neurosurg Psychiatry. 1999;67(2):234-238.

9. Fischer A, Zalvide J, Faurobert E, et al. Cerebral cavernous malformations: from CCM genes to endothelial cell homeostasis. Trends Mol Med. 2013;19(5):302-308.

10. Guclu B, Ozturk AK, Pricola KL, et al. Cerebral venous malformations have distinct genetic origin from cerebral cavernous malformations. Stroke. 2005;36(11):2479-2480.

11. Hon JM, Bhattacharya JJ, Counsell CE, et al. The presentation and clinical course of intracranial developmental venous anomalies in adults: a systematic review and prospective, population-based study. Stroke. 2009;40(6):1980-1985.

\section{Disclosures}

Dr. Steinberg is a consultant for Qool Therapeutics, NeuroSave, SanBio, and Zeiss. He is a patent holder with Peter Lazic, US.

\section{Author Contributions}

Conception and design: Grant, Prolo, Loven, Edwards, Steinberg. Acquisition of data: Prolo, Vogel. Analysis and interpretation of data: Grant, Prolo, Jin. Drafting the article: Prolo, Jin. Critically revising the article: Grant, Prolo, Jin, Edwards, Steinberg. Reviewed submitted version of manuscript: Grant, Prolo, Jin, Loven, Steinberg. Statistical analysis: Jin. Study supervision: Grant, Prolo, Edwards, Steinberg.

\section{Supplemental Information Online-Only Content}

Supplemental material is available with the online version of the article.

Supplemental Table 1. https://thejns.org/doi/suppl/10.3171/ 2020.2.PEDS19543.

\section{Correspondence}

Gerald A. Grant: Stanford University School of Medicine, Stanford,CA.ggrant2@stanford.edu. 